\title{
3D Structure from Jlab12 to EIC
}

\author{
Harut Avakian * \\ Jefferson Lab \\ E-mail: avakian@jlab.org
}

Understanding the dynamics of partons in general, and non perturbative sea, in particular, will be crucial for the understanding of strong interactions. Correlations of the spin of the target or/and the momentum and the spin of quarks, combined with final state interactions define the azimuthal distributions of produced particles. Production of correlated hadron pairs, in addition, will play an increasingly important role in the interpretation of pion electroproduction data. Studies of the nucleon structure beyond the traditional leading twist and current fragmentation, provide qualitatively new tools to study the nucleon structure and extension of large $x$ region well covered by JLab, to large $Q^{2}$ at EIC will be very important in interpretation of the data from JLab. The large acceptance of the EIC combined with clear separation of target and current fragmentation regions will also provide a unique possibility to study the nucleon structure in the target fragmentation region and correlations of target and current fragmentation regions.

23rd International Spin Physics Symposium - SPIN2018 -

10-14 September, 2018

Ferrara, Italy

\footnotetext{
${ }^{*}$ for the CLAS Collaboration
} 


\section{Introduction}

Measurements of spin and azimuthal asymmetries in various processes in recent years have stimulated theoretical interest and progress in studies of the nucleon structure. Interpretation of experimental data in terms of parton distribution functions, generalized to describe transverse momentum and spatial parton distributions, is one of the main remaining challenges of modern nuclear physics. These new parton distribution and fragmentation functions encode the motion and the position of partons and are often referred to as three-dimensional distributions describing the three-dimensional (3D) structure of the nucleon. Understanding of the production mechanism and performing phenomenological studies compatible with factorization theorems using minimal model assumptions are goals of analyses of the experimental data. The data on Semi-Inclusive Deep Inelastic Scattering (SIDIS) played a crucial role in the current understanding of nucleon spin-phenomena. The Jefferson Lab $12 \mathrm{GeV}$ upgrade data on polarized and unpolarized SIDIS will have remarkably higher precision at large parton fractional momentum $x$ compared to the existing data. Transition from simple, one dimensional description using collinear parton distributions that depend on nucleon's longitudinal momentum fraction, $x$, to more complex nucleon picture with interacting and orbiting quarks, leads to the introduction of Transverse Momentum Dependent (TMD) partonic distributions. SIDIS provides access to TMD partonic distributions through measurements of spin and azimuthal asymmetries. Several experiments worldwide were involved in studies of SIDIS with different hadrons produced. Most of the latest relevant data for studies of spin-orbit correlations was coming from the HERMES Collaboration at HERA, the COMPASS Collaboration at CERN, and measurements already performed at JLab. As for the near future, a wide spectra of high-precision measurements will be done at JLab12 [1], while COMPASS plans to collect more SIDIS data with transversely polarized deuteron target in 2021 [2]. Wider angle coverage of CLAS12 detector allows measurements in a wide range of $P_{h T}$ (up to $1.5 \mathrm{GeV}$ ), and $Q^{2}$ (up to $10 \mathrm{GeV}^{2}$ ), while the SoLID detector would allow measurements of all kind of polarization asymmetries at large Bjorken- $x$ with superior precision.

At JLab all 3 halls are involved in 3D structure studies [3] including the HMS and Super HMS at Hall C [4, 5, 6], the BigBite and Super BigBite, as well as, the SoLID detector at Hall A [7, 8, 9], and CLAS12 at Hall B $[10,11]$. Several experiments are already approved to study in details the azimuthal modulations in SIDIS for different hadron types, targets, and polarizations in a broad kinematic range $[10,11,12,13,8,5,7,9]$. The experimental investigation of medium modification of quark fragmentation and spin-orbit correlation will be also extensively pursued at the upgraded Jefferson Lab facility, for which several related experimental proposals already exist [14, 15].

Studies of spin-azimuthal asymmetries in semi-inclusive and hard exclusive production of photons and hadrons have been widely recognized as key objectives of the Jefferson Lab $12 \mathrm{GeV}[16,3]$ upgrade and one of the driving forces for the future Electron Ion Collider [17, 18]. The spin and orbital structure of the hadrons was always challenging for the theory, as quark-gluon interactions, and their correlations with the spin of partons and hadrons, are very significant and often are not easily understood in a simple picture of a static partons in the nucleon. Challenges in interpretation of data from inclusive hadron SIDIS experiments, which play a dominant role in studies of nucleon spin-orbital structure were discussed in details in recent review [19]. Most prominent phenomena to address, are the different background contributions from target fragmentation and 
correlations between target and current fragmentation regions, correlated di-hadron production, exclusive hadron production, higher twist contributions, and limitation of phase space of experiments. Precision studies of SIDIS observables also require detailed understanding of transverse momentum dependence of fragmentation functions and QED radiative corrections mixing contributions from different structure functions. Studies of SIDIS involving polarized nuclear targets, in addition require detailed understanding of nuclear modifications of orbital motion of partons. The development of a comprehensive extraction framework will be crucial to facilitate extraction of 3D nucleon structure, helping to understand various assumptions in the extraction and data analysis, also helping to insure the model independence of the interpretation of the experimental data and validate the extracted underlying non-perturbative functions.

\section{Spin-Azimuthal distributions}

The SIDIS $\left(\ell(k)+N(P) \rightarrow \ell^{\prime}\left(k^{\prime}\right)+h\left(P_{h}\right)+X\left(P_{X}\right)\right)$ reaction is such that a beam lepton $\ell$ with the 4-momenta $k$, scatters off of a target nucleon, $N$ with four momentum $P$, and the scattered lepton $\ell^{\prime}$ with four momentum $k^{\prime}$ is detected along with a single hadron, $h$ with four momentum $P_{h}$; all other produced particles in the final state, $X$, are not detected. Assuming a single photon exchange, the SIDIS cross-section can be decomposed into a sum of various azimuthal modulations coupled to corresponding structure functions. SIDIS cross section has following form [20, 21, 22]:

$$
\begin{aligned}
& \frac{d \sigma}{d x d y d z d P_{h T}^{2} d \phi_{h} d \phi_{S}}=\hat{\sigma}_{U}\left\{1+\varepsilon A_{U U}^{\cos 2 \phi_{h}} \cos 2 \phi_{h}+\sqrt{2 \varepsilon(1+\varepsilon)} A_{U U}^{\cos \phi_{h}} \cos \phi_{h}\right. \\
& +\lambda_{\ell} \sqrt{2 \varepsilon(1-\varepsilon)} A_{L U}^{\sin \phi_{h}} \sin \phi_{h} \\
& +S_{\|}\left[\sqrt{2 \varepsilon(1+\varepsilon)} A_{U L}^{\sin \phi_{h}} \sin \phi_{h}+\varepsilon A_{U L}^{\sin 2 \phi_{h}} \sin 2 \phi_{h}\right] \\
& +S_{\|} \lambda_{\ell}\left[\sqrt{1-\varepsilon^{2}} A_{L L}+\sqrt{2 \varepsilon(1-\varepsilon)} A_{L L}^{\cos \phi_{h}} \cos \phi_{h}\right] \\
& +S_{\perp}\left[A_{U T}^{\sin \left(\phi_{h}-\phi_{S}\right)} \sin \left(\phi_{h}-\phi_{S}\right)+\varepsilon A_{U T}^{\sin \left(\phi_{h}+\phi_{S}\right)} \sin \left(\phi_{h}+\phi_{S}\right)+\varepsilon A_{U T}^{\sin \left(3 \phi_{h}-\phi_{S}\right)} \sin \left(3 \phi_{h}-\phi_{S}\right)\right. \\
& \left.+\sqrt{2 \varepsilon(1+\varepsilon)} A_{U T}^{\sin \phi_{S}} \sin \phi_{S}+\sqrt{2 \varepsilon(1+\varepsilon)} A_{U T}^{\sin \left(2 \phi_{h}-\phi_{S}\right)} \sin \left(2 \phi_{h}-\phi_{S}\right)\right] \\
& +S_{\perp} \lambda_{\ell}\left[\sqrt{\left(1-\varepsilon^{2}\right)} A_{L T}^{\cos \left(\phi_{h}-\phi_{S}\right)} \cos \left(\phi_{h}-\phi_{S}\right)\right. \\
& \left.\left.+\sqrt{2 \varepsilon(1-\varepsilon)} A_{L T}^{\cos \phi_{S}} \cos \phi_{S}+\sqrt{2 \varepsilon(1-\varepsilon)} A_{L T}^{\cos \left(2 \phi_{h}-\phi_{S}\right)} \cos \left(2 \phi_{h}-\phi_{S}\right)\right]\right\}
\end{aligned}
$$

where asymmetries $A_{\ldots}^{\ldots}$ [22] depend on kinematical variables $x, Q^{2}, z, P_{h T}$ and correspond to azimuthal modulations of the cross section in the azimuthal angle $\phi_{S}$ of transverse spin and/or azimuthal angle $\phi_{h}$ of the produced hadron, both defined in the $\gamma^{*} N$ Center of Mass (CM) frame. The first and second subscripts denote respectively the lepton and target nucleon polarizations, while the superscript indicates the corresponding azimuthal modulation. Asymmetries are defined as ratios of corresponding polarized structure functions $F_{\ldots}^{\ldots}$ and unpolarized structure function $F_{U U}$ The unpolarized structure function, $F_{U U}$, or more precisely combination of structure functions corresponding to transverse and longitudinal polarization of the virtual photon $F_{U U, T}+\varepsilon F_{U U, L}$, is included in the definition of $\hat{\sigma}_{U}$. The $\lambda_{\ell}, S_{\|}$and $S_{\perp}$ are polarizations of the incoming lepton, and 
longitudinal and transverse polarizations of the nucleon, respectively. We use the usual SIDIS kinematical variables $x, y$, and $z$ defined as: $x=Q^{2} / 2(P \cdot q), y=(P \cdot q) /(P \cdot k), z=\left(P_{h} \cdot P\right) /(P \cdot q)$, where $Q^{2}=-q^{2}=-\left(k-k^{\prime}\right)^{2}$ is the negative four-momentum squared of the virtual photon, and $P_{h T}$ is the transverse momentum of the detected hadron. The ratio $\varepsilon$ of the longitudinal and transverse photon flux is given by: $\varepsilon=\frac{1-y-\gamma^{2} y^{2} / 4}{1-y+y^{2} / 2+\gamma^{2} y^{2} / 4}$, where $\gamma=2 M x / Q$, and $M$ is the mass of the nucleon.

In the kinematical region, where the TMD description of SIDIS is appropriate, namely in the beam fragmentation region, $P_{h T} / z \ll Q$, the transverse momentum of the produced hadron $P_{h T}$ is generated by intrinsic momenta of the parton in the nucleon, $\mathbf{k}_{T}$, and the transverse momentum of the produced hadron with respect to the fragmenting parton, $\mathbf{p}_{T}$, such that the structure functions become convolutions of TMD parton distribution functions (PDFs), and TMD fragmentation functions (FFs). The convolution integral, for a given combination of TMD PDF $f$ and FF $D$ reads [22]

$$
\mathscr{C}[w f D]=x \sum_{q} e_{q}^{2} \int d^{2} \mathbf{k}_{T} d^{2} \mathbf{p}_{T} \boldsymbol{\delta}^{(2)}\left(\mathbf{p}_{T}+z \mathbf{k}_{T}-\mathbf{P}_{h T}\right) w\left(\mathbf{k}_{T}, \mathbf{p}_{T}\right) f^{q}\left(x, k_{T}^{2}\right) D^{q}\left(z, P_{h T}^{2}\right),
$$

where $w$ is a kinematical factor, and the sum goes over all flavors of quarks and anti-quarks. Well known SIDIS structure functions $F_{U U, T}$ and $F_{L L}$ will be, thus, described by convolutions of $f_{1}$ and $g_{1}$ TMD PDFs and $D_{1}$ the unpolarized TMD fragmentation function, with $F_{U U, T}=\mathscr{C}\left[f_{1} D_{1}\right], F_{L L}=$ $\mathscr{C}\left[g_{1} D_{1}\right]$. The TMDs depend on polarization state of the quark (rows) and polarization state of the nucleon (columns). The corresponding tables for TMD FFs can be found in Review [23].

Many structure functions involve transversely polarized quarks. For example the $F_{U U}^{\cos 2 \phi}$, at leading twist is interpreted as a convolution of Boer-Mulders distribution function, $h_{1}^{\perp}$ that encodes the correlation between the transverse motion of a quark and its own transverse spin [24], and the Collins fragmentation function, $H_{1}^{\perp}$ [25], that describe fragmentation of transversely polarized quarks into an unpolarized hadron.

One of the most important questions about the 3D structure of the nucleon are the $\mathbf{k}_{T}$ and $\mathbf{p}_{T}$ shapes of the distribution and fragmentation TMDs and flavour and spin dependence of those shapes. For precision studies of TMDs it is also important to understand the role of medium, and the effects of in medium modifications of TMDs. That is crucial, since both COMPASS and JLab use nuclear targets to study polarization effects. Another important question to address is the role of exclusive processes in studies of SIDIS. In order to extract underlying functions and thus details of dynamics of quarks and gluons from SIDIS data one also has to have a good understanding of the underlying fragmentation process in which quark fragments into an observed hadron. Exclusive processes may shed light on the fragmentation process itself.

The most prominent leading twist observable is the $\phi_{h}$-integrated cross section described by the $F_{U U}$ structure function. Experiments, however, prefer to measure the multiplicities of hadrons, which is the ratio of SIDIS cross sections for a given type of hadron divided by DIS cross section in a given bin in $x, Q^{2}$, (the advantage is that e.g. the scattered lepton acceptance entering in the numerator and denominator cancels).

Collinear PDFs have flavour dependence, thus it is not unexpected that also the transverse momentum dependence may be different for the different flavours [26]. Model calculations of transverse momentum dependence of TMDs [27, 28, 29, 30] and lattice QCD results [31, 32] suggest that the dependence of widths of TMDs on the quark polarization and flavor may be significant. 
It was found, in particular, that the average transverse momentum of antiquarks is considerably larger than that of quarks [33,34]. The frequently used assumption of factorization of $x$ and $k_{T}$ (or $z$ and $P_{h T}$ ) dependencies [35] may be significantly violated (see Fig. 10 of [36]). For instance the predicted average transverse momentum square $\left\langle k_{T}^{2}\right\rangle$ of quarks and antiquarks may depend strongly on their longitudinal momentum fraction $x$ within the framework of the chiral quark soliton model. Comparison of COMPASS with HERMES and JLab measurements of multiplicities performed in Ref. [37] revealed significant differences which could be due to the different $Q^{2}$ ranges covered by the experiments and supports findings of studies from Ref. [38] indicating that at lower energies the large values of $P_{h T}$ are suppressed due to smaller phase space, in particular at large $z$. The latter is confirmed by recent COMPASS results obtained for the $\mathrm{K}^{-}$over $\mathrm{K}^{+}$multiplicity ratio at large fraction $z$ of the virtual-photon energy [39].

Measurements performed with polarized nuclear targets $\left(\mathrm{NH}^{3}\right)$, require detailed account of significant nuclear background, and very careful treatment is needed to estimate the dilution factor, which defines the fraction of events originating from polarized quasi free protons. The double-spin asymmetries in DIS and $\pi^{0}$ SIDIS, in simple parton model, at large $x$, where the sea contribution is negligible, are expected to be roughly the same. CLAS measurements of both asymmetries indicate that already at $6 \mathrm{GeV}$, they are in good agreement [40].

Precision measurements using the upgraded CLAS12 detector with polarised $\mathrm{NH}_{3}$ and $\mathrm{ND}_{3}$ targets will allow to access the $k_{T}$-distributions of $u$ and $d$-quarks aligned and anti-aligned with the spin of the nucleon. Projections for the resulting $P_{h T}$-dependence of the double spin asymmetries for all three pions are shown in Fig. 1 for a $\mathrm{NH}_{3}$ target [11, 13]. Integrated over transverse momentum, the data will also be used to extract the $k_{T}$-integrated standard PDFs. Two proposals have been approved to study SSAs with a longitudinally polarised target using the SoLID detector [9] and Super-Bigbite spectrometer with polarised ${ }^{3} \mathrm{He}$ targets [7]. The later one will provide kaon identification using a RICH detector.

Large $\sin \phi$ SSA measurements by HERMES [41] spawned a number of additional measurements of SSAs and DSAs (Double Spin Asymmetries) using polarized hydrogen and deuterium targets $[42,43]$. Most prominent are the Collins and Sivers asymmetries. The study of the Sivers effect, describing correlations between the transverse polarization of the nucleon and its constituent (unpolarized) parton's transverse momentum, has been the topic of intensive experimental, phenomenological and theoretical effort in recent years. From the comparison of HERMES [44] and COMPASS [45] proton results in the overlapping kinematic region, unlike the Collins asymmetry, the Sivers effect at HERMES was found to be somewhat larger compared to that measured at COMPASS. This observation may indicate the influence of TMD evolution effects. Evolution properties of TMDs and in particular the Sivers TMD, were predicted to be very different from regular PDFs [46]. Present models predict for increasing $Q^{2}$ a slight dependence of the Sivers asymmetry for DGLAP and a stronger dependence for different TMD evolution schemes. In order to test this conjecture, COMPASS recently performed the first multi-differential analysis of the transverse-spin-dependent asymmetries extracting them from SIDIS data at four-five different hard scales [47, 48, 49]. Extracted $Q^{2}$-dependences of the Sivers SSAs in different bins of $x$ were fitted with a linear decreasing function and a constant with a slight statistical preference for the former case.

Studies of evolution of Sivers TMD require precision measurements in different ranges of $Q^{2}$. 
A projection for $Q^{2}$-dependence of the Sivers effect expected from CLAS12 is shown in Fig. 1. The asymmetry, however, as other observables which are constructed by taking ratios, are not ideal grounds for the study of TMD evolution effects, as it has additional modulations coming from the unpolarized part, making interpretation more complicated. Due to partial cancellation of evolution effects in numerator and denominator, the asymmetries themselves may exhibit only a weak $Q^{2}$-dependence. It was suggested that more effort should be made towards measuring properly normalized SIDIS and e+e-, and Drell-Yan cross sections (both unpolarised and polarised).
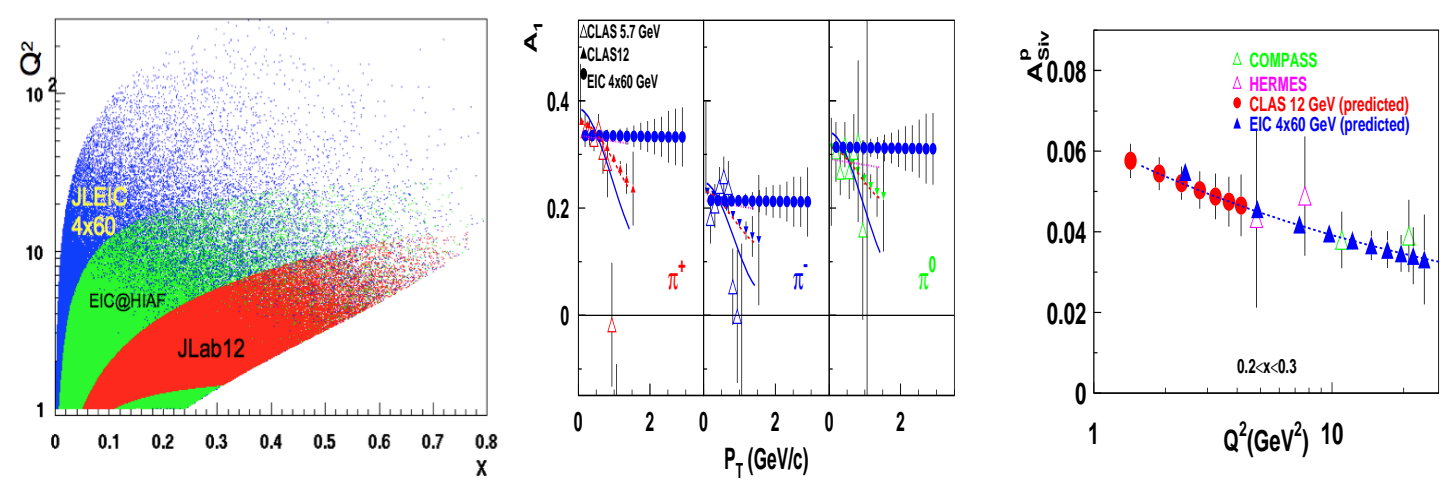

Figure 1: Kinematical coverage of JLab12 and two options of EIC (left) and projections for CLAS12 measurements of double-spin asymmetry $A_{1}$ for pion production as a function of $P_{h T}$ compared to published data from CLAS [50], and $Q^{2}$-dependence of the Sivers asymmetry for $e p \rightarrow e^{\prime} \pi^{+} X$, compared to HERMES, COMPASS and future EIC measurements using the EIC[51] configuration with $4 \mathrm{GeV}$ electrons and $60 \mathrm{GeV}$ protons (100 days at $\left.10^{34} \mathrm{~cm}^{-2} \mathrm{sec}^{-1}\right)$ [13].

The measurements of the SSAs for hadrons produced in the Target Fragmentation Region (TFR) will allow one to study the structure of the nucleon through the fracture functions. These objects, though more complicated than the ordinary PDFs and FFs, will provide important new information. An updated version of the PYTHIA, mPYTHIA was used to look at correlations between different kinematical regions [52]. Lund string model used in PYTHIA differs from the usual QCD factorized approach that describes the hadron production in the Current Fragmentation Region (CFR) with a convolution of PDFs and FFs and in the TFR using two additional independent fracture functions. Studies based on mPYTHIA, accounting only the correlation between the nucleon's transverse polarization and the transverse momentum of the struck quark revealed sizable signal in the in TFR, comparable in size to that in the CFR. Experimental measurements of Sivers SSAs in both CFR and TFR will be important to reveal underlying correlations.

\section{Higher twist observables}

Most important observables sensitive to TMDs are the $Q^{2}$ and $P_{h T}$ dependences of final cross sections, providing access to evolution and transverse momentum dependence of TMDs. Apart from the $Q^{2}$ dependence of the elementary lepton-quark cross section $\propto Q^{-4}$, structure functions 
appearing in the cross section also change with hard scale $Q$. Higher twist structure functions will include convolutions of higher twist TMD functions. The higher twist non-perturbative functions describe various spin-spin and spin-orbit correlations as corresponding operators include additional gluon and/or quark fields in the matrix element. Recent studies of higher twist distributions in various models $[53,54]$ and in lattice QCD $[55,56,57]$ demonstrated that those functions can be very significant, contradicting popular assumptions that higher twists terms are small. Indeed, while $F_{L U}^{\sin \phi_{h}}$ is expected to vanish in Wandzura-Wilczek-type approximation, experiments observe very significant $\sin \phi$ modulations $[58,59]$.

Subleading structure functions require a proof of validity of TMD factorization at higher twist and the proof is not yet available, however studies of sub-leading twists are also important, as they may affect significantly the extraction of leading-twist moments, as the radiative effects, and complicated acceptances of wide angle spectrometers may introduce cross-talk between different azimuthal moments in the spin dependent and spin-independent moments. For instance $\cos \phi$ modulation asymmetry known as the Cahn effect [60], is significant $\sim 20 \div 30 \%$ and dominating in the $P_{h T} \sim 1 \mathrm{GeV}$ range, even though it is suppressed by $P_{h T} / Q$ with respect to leading twist asymmetries. Higher twist asymmetries, especially those not receiving contributions from leading twist structure functions, were measured and in most of the cases were found not only to be not compatible with zero, but very significant. Good examples are the $\cos \phi_{h}$ moment of the unpolarized cross section, $F_{U U}^{\cos \phi_{h}}$, first measured by the EMC collaboration, back in the 80's [61, 62]. The azimuthal modulations have been studied phenomenologically, for instance Ref. [63] investigated effects due to the phase space limitations due to finite beam energies of real experiments. The cosine modulations, in particular, are very sensitive to the corrections due to limitation of the phase space in experiments. The twist-3 nature of $\cos \phi_{h}$ modulations could be tested by examining their $Q^{2}$ dependence.

Other important contributions include the $\sin \phi_{h}$ moment depending on the longitudinally target polarization, $F_{U L}^{\sin \phi_{h}}$, first measured by the HERMES collaboration in the $90 \mathrm{~s}[64,41,42,43]$, and the $\sin \phi_{h}$ moment depending on the longitudinal polarization of the beam, $F_{L U}^{\sin \phi_{h}}$, first measured at JLab $[58,65,66]$. All those measurements were repeated and confirmed later by HERMES, COMPASS and JLab. Since the structure functions $F_{L U}$ and $F_{U L}$ contain kinematical terms depending on the beam energy for given kinematics, as well as an additional $1 / Q$ suppression factor, direct comparison of ratios of structure functions involved in those observables between different experiments requires accounting for those terms. After corrections the data seem to be consistent also between CLAS and HERMES $A_{L U}^{\sin \phi_{h}}$. Recent high precision measurements of $A_{U L}^{\sin \phi_{h}}$ performed at COMPASS are also consistent with similar measurements at HERMES. Both asymmetries exhibit similar kinematical behaviour and, more importantly similar flavor dependence. Accounting for difference in energies and average $y$ in all comparisons was done by dividing by the kinematic factors defined in Eq.2.1 (for $A_{U L}^{\sin \phi_{h}}$ the factor $F(y)=\sqrt{2 \varepsilon(1+\varepsilon)}$ ). Latest measurements of $A_{U L}^{\sin \phi_{h}}$ by CLAS [40] for all pion flavors is consistent with HERMES measurements, confirming that $\pi^{+}$ and $\pi^{0}$ show similar behaviour both for $A_{L U}^{\sin \phi_{h}}$ and $A_{U L}^{\sin \phi_{h}}$.

A significantly higher, compared to JLab12, $P_{h T}$ range accessible at EIC would allow for studies of transverse momentum dependence of various distribution and fragmentation functions as well as transition from TMD regime $\left(P_{h T} / z \ll Q\right)$ to collinear perturbative regime $\left(P_{h T} / z \sim\right.$ $Q)$. Measurements of spin and azimuthal asymmetries as a function of the final hadron transverse 
momentum at EIC will extend (see Fig. 2) measurements at JLab12 [67] to significantly higher $P_{h T}$ and lower $x$ and will provide access to studies of TMDs beyond the valence region. A much higher $Q^{2}$ range accessible at EIC would allow for studies of the $Q^{2}$-dependence of different higher twist spin-azimuthal asymmetries (Fig 2), which, apart from providing important information on quark-gluon correlations are needed for understanding of possible corrections from higher twists to leading twist observables.
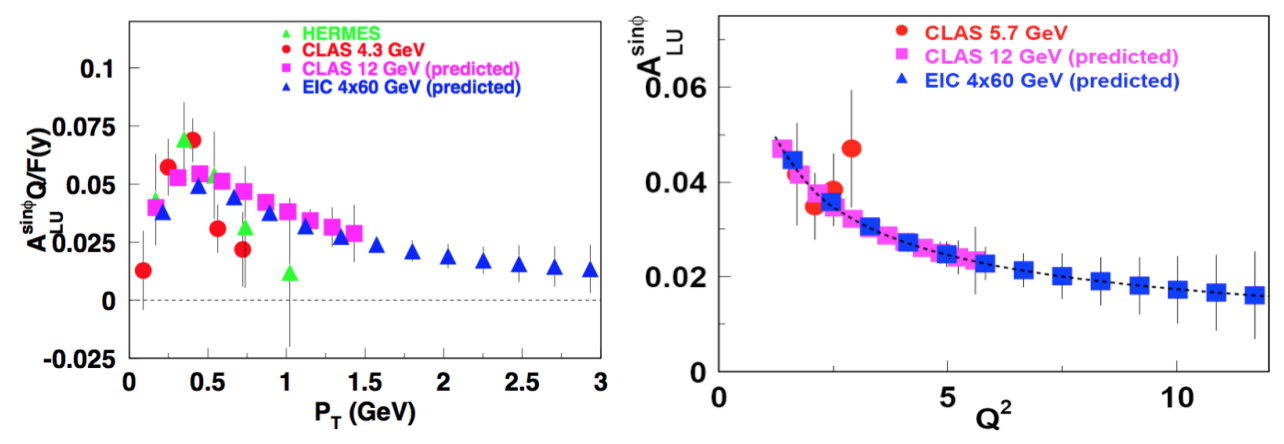

Figure 2: Projections for higher-twist lepton spin asymmetry $A_{L U}^{\sin \phi}$ for positive pion production, using 4 $\mathrm{GeV}$ electrons and $60 \mathrm{GeV}$ protons (100 days at $10^{34} \mathrm{~cm}^{-2} \mathrm{sec}^{-1}$ ), as a function of $P_{h T}$ (left) and $Q^{2}$ (right) compared to published data from CLAS [58] and HERMES [59] and projected CLAS12 [67] in one $x, z$ bin $(0.2<x<0.3,0.5<z<0.55)$.

\section{Dihadron production}

Hadron pair production $\ell(l)+N(P) \rightarrow \ell\left(l^{\prime}\right)+H_{1}\left(P_{1}\right)+H_{2}\left(P_{2}\right)+X$, was first proposed for extraction of transversity distribution in a complementary to single hadron production case and was used in extraction of transversity in an analysis with combined electron-proton and proton-proton data [68].

The interpretation of di-hadron production, as well as interpretation of single-hadron production is intimately related to contributions to those samples from vector mesons. The general procedure for SIDIS analysis, so far, was requiring estimates of contribution of diffractive $\rho^{0}$, so theoretical studies can account for their contribution. Since the spin dependent fragmentation (Collins function) of rho mesons is very different from pions, in fact predicted to have an opposite sign [69], the final interpretation of pion asymmetries will be very sensitive to relative fraction of pions coming from vector meson decays. Large discrepancies between perturbative calculations in the kinematical region $P_{h T} / z>Q$ reported in Ref. [70] may be well related to significant fraction of decay pions, produced by correlated di-hadrons produced in the region of small $P_{h T} / z$ [19]. The PYTHIA based MC, suggests that the dominating fraction of pions are indeed coming from vector meson decays (see Fig.3). Measurements of SSA performed by CLAS indicate that there is a significant asymmetry in the single-pions sample originating from rhos, which is also very different for different vector mesons. The size of the asymmetry reaches $\sim 20 \%$ and is opposite for $\pi^{+}$ originating from exclusive $\rho^{+}$and $\rho^{0}$ decays [71].

Significant single beam spin asymmetries were predicted in di-hadron production for several angular correlations. The usual $\sin \left(\phi_{R}\right)$ single beam spin asymmetry [72] involves subleading- 

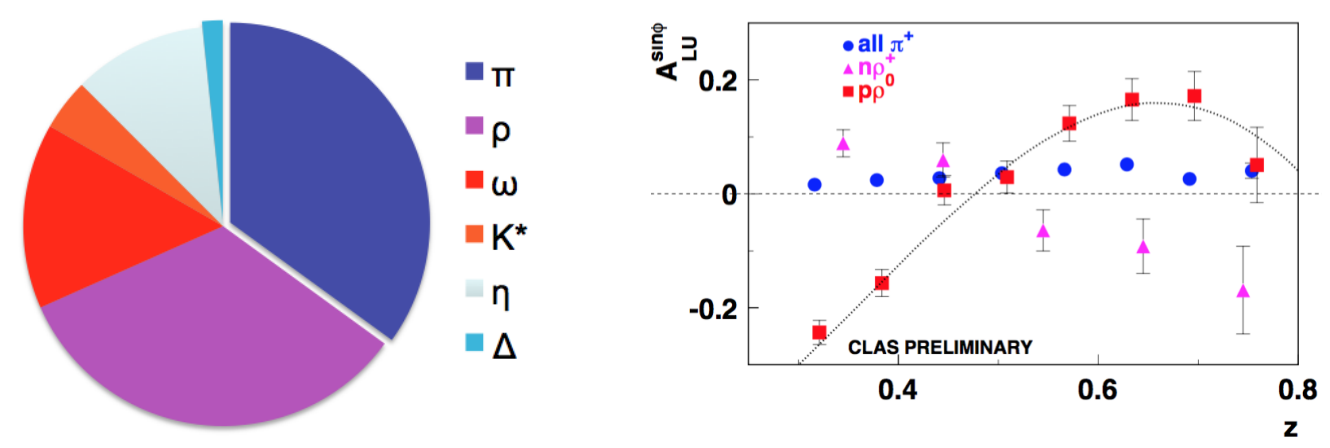

Figure 3: Left panel: The fraction of pions in $e p \rightarrow e^{\prime} \pi X$ in HERMES kinematics (27.5 GeV), produced directly from the string in PYTHIA MC (shown as $\pi$ ) and from different decay channels $\left(\rho, \omega, K^{*}, \eta\right.$ and $\Delta)$. Right panel: Measured by CLAS $A_{L U}^{\sin \phi}$ on hydrogen target, for final state $\pi^{+}$originating from exclusive $\pi^{+} \pi^{-}$events dominated by $\rho^{0}$ (red squares), from exclusive $\pi^{+} \pi^{0}$ events dominated by $\rho^{+}$(magenta triangles) and integrated over all sources (blue circles) [71]. The curve shows the expectations for $\pi^{+} A_{L U}^{\sin \phi}$ from MC for exclusive $\rho$ s generated with measured $A_{L U}^{\sin \phi}$.

twist functions in the so-called "collinear kinematics" that involves the measurement of only the relative transverse momenta of the hadron pair. Recently, a new leading-twist single beam spin asymmetry involving the helicity-dependent $\mathrm{DiFF} G_{1}^{\perp}$ has been proposed [73], in analogy with the target single spin asymmetry in Refs. [74], which requires the measurement of both total and relative transverse momenta of the pair. Yet another leading twist beam spin asymmetry involves the so-called fracture functions [75], where one of the hadrons is produced in the current region by the fragmenting parton, while the second one is produced in the target region by the target remnant. CLAS preliminary measurements [76] indicated a very significant non-zero beam-spin asymmetry $A_{L U}$ both on ${ }^{2} \mathrm{H}$ and $\mathrm{NH}_{3}$ targets. Non zero single-spin asymmetries $\left(A_{L U}\right)$ have also been observed in back-to-back (b2b) pion and proton electroproduction [77], indicating that spinorbit correlations may be very significant. While this opens a new avenue for studies of the complex nucleon structure in terms of quark and gluon degrees of freedom, it also suggests, that there are likely no uncorrelated hadrons in SIDIS.

The production of two hadrons in polarized SIDIS, with one spinless hadron produced in the current fragmentation region and another in the TFR, would provide access to the target-current correlations, described by leading twist FFs $[78,75]$.

The beam spin asymmetry, $A_{L U}^{\sin \Delta \phi}$, has been calculated in that formalism as a sinusoidal modulation of the difference of azimuthal angles of proton and $\pi^{+}$with respect to the lepton scattering plane, for different electron helicity states. The modulation was extracted for different bins in $x$, $z$ of the pion and the product of transverse momenta of final state proton and pion with respect to the virtual photon in the $\mathrm{CM}$ frame. The difference in counts of positive and negative electron helicities indeed exhibit a sinusoidal behaviour in $\Delta \phi$. Above the kinematic region where the target fragment may be an exclusive Delta $\left(M_{X}>1.5 \mathrm{GeV}\right)$ all three pions have similar behavior, indicating there is a strong correlation between hadrons produced in the current and target fragmentation regions. The $P_{T}$-dependence of the SSA (see Fig. 4) shows a trend for the asymmetry to increase with increasing transverse momenta of pion and proton $P_{T 1}, P_{T 2}$, consistent with expectations from theory. The $x$-dependence of the the $A_{L U}^{\sin \phi}$ has been found to be consistent with asymmetry being 
large in the large- $x$ region, were the valence quark presence is very significant.
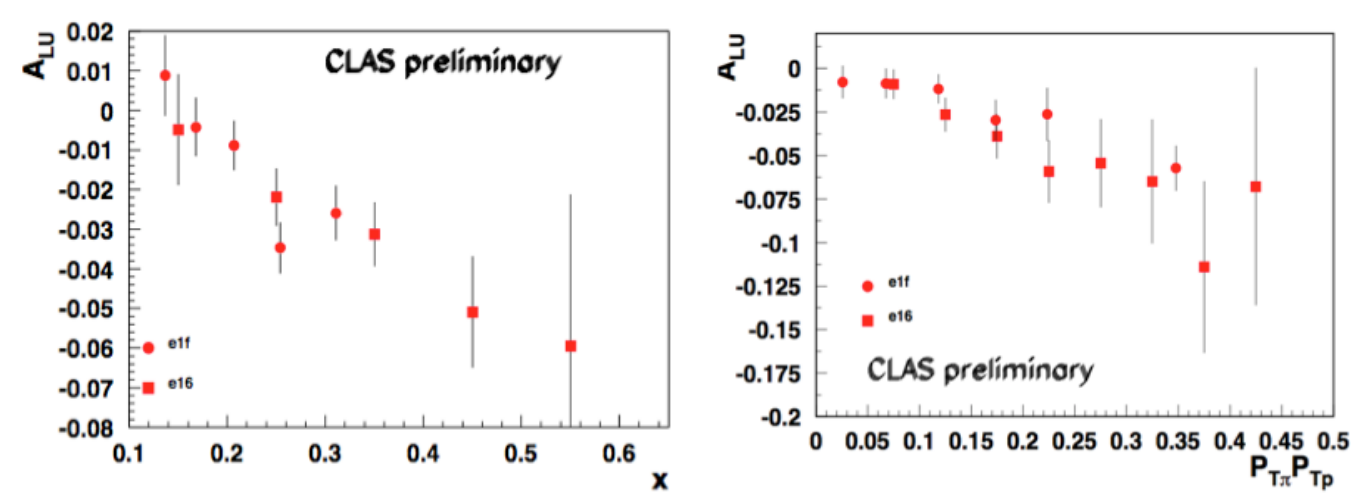

Figure 4: $A_{L U}$ dependence on $x$ and the product of transverse momenta of the pion and proton in $\mathrm{GeV}^{2}$, for $\pi^{+}$.

For quantitative comparison with ongoing measurements as well as projections for future measurements of different $\mathrm{b} 2 \mathrm{~b}$ processes using the CLAS12 and EIC one will need modeling of FFs, which can be modeled using different partonic models used to predict polarization of $\Lambda$ hyperons in the target fragmentation region of DIS, such as the meson cloud model [79] or intrinsic strangeness model for $\Lambda$ production in the target fragmentation region in deep-inelastic scattering [80, 81].

Most of the Lambdas, even at EIC energies, are produced in the target fragmentation region (see Fig. 5) at negative $x_{F}$, with only fraction of them produced in the current fragmentation, where their counts should be comparable to anti-Lambdas.
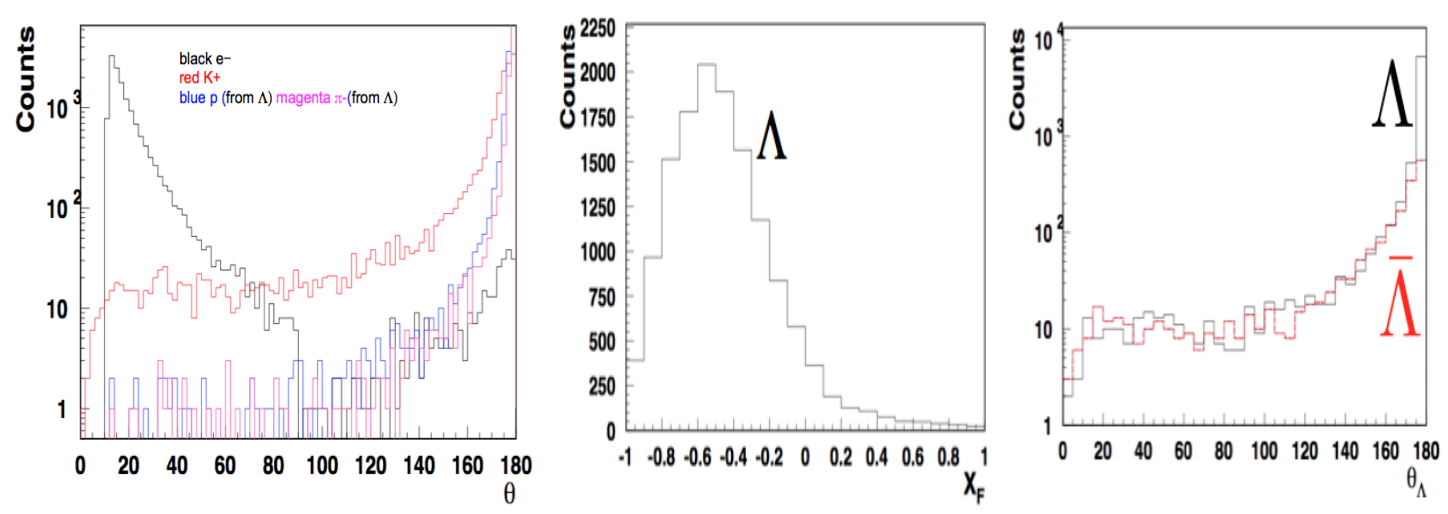

Figure 5: Angular distributions of particles (left), distribution of Lambdas vs fraction of the total longitudinal momentum in the CM frame carried by the Lambda, $x_{F}$, (middle) and distribution of Lambdas and anti-Lambdas versus the production angle in the Lab frame (right panel).

\section{Summary}

Although the interest in TMDs has grown enormously, we are still in need of a consistent theoretical and phenomenological description spanning the full kinematic regime covered by the (un)polarized world-data. Some TMDs have been already phenomenologically extracted, mainly 
from analyzing azimuthal distributions of single hadrons in SIDIS. To obtain a full picture about the 3D momentum structure of the partons in the nucleon from high to low $x$, it is important to connect the theoretical approaches to extract TMDs including evolution. The studies of 3D PDFs in general, and TMDs in particular, require a lot more attention to uncertainties due to input parametrizations, as more degrees of freedom and bigger number of input parameters may generate biased model uncertainties.

Measurements of di-hadron single spin asymmetries indicate that final state hadrons tend to be correlated. Several effects, including limited phase space for finite beam energies, higher twist contributions, radiative corrections and possible background from target fragmentation region should be considered as important elements for interpretation of systematic uncertainties in precision measurements in polarized SIDIS experiments, and in particular experiments at Jefferson Lab. Understanding of the scale of contributions $\left(\sim M^{2} / Q^{2}, \sim P_{h T}^{2} / Q^{2}\right.$, Target/Current correlations, etc) will define the limits on precision needed to various aspects of TMDs, such as evolution, higher twists, etc. Sophisticated frameworks for calculation of 3D PDFs, such as TMDlib [82] can be interfaced to extend the scope of available models, which can be used in validation of 3D PDFs extraction framework. The future Extraction and VAlidation framework [19] will serve to help both experimental community and phenomenological community to test results and assure model independence of interpretation of measured data.

\section{Acknowledgements}

This work was supported by the U.S. Department of Energy under Contract No. DE-AC0506OR23177.

\section{References}

[1] V.D. Burkert, Ann. Rev. Nucl. Part. Sci. 68 (2018) 405.

[2] COMPASS, K. Augsten et al., SPSC-P-340-ADD-1, CERN-SPSC-2017-034 (2017).

[3] J. Dudek et al., Eur. Phys. J. A48 (2012) 187, arXiv:1208.1244.

[4] R. Ent et al., JLab Experiment E12-06-104 (2006).

[5] R. Ent et al., JLab Experiment E12-09-017 (2009).

[6] R. Ent et al., JLab Experiment E12-13-007 (2013).

[7] G. Cates et al., JLab Experiment E12-09-018 (2009).

[8] H. Gao, Q. X. and P. J.-C., JLab Experiment E12-10-006 (2008).

[9] J.P. Chen et al., JLab Experiment E12-11-007 (2008).

[10] H. Avakian et al., JLab Experiment E12-06-112 (2006).

[11] H. Avakian et al., JLab Experiment E12-07-107 (2007).

[12] H. Avakian et al., JLab Experiment E12-09-008 (2009).

[13] H. Avakian et al., JLab Experiment E12-09-009 (2009).

[14] H. Avakian, JLab LoI LOI-12-14-004 (2014). 
[15] W. Brooks and S. Kuhn, JLab Experiment E12-14-001 (2014).

[16] A. Prokudin, Phys. Part. Nucl. 44 (2013) 947, arXiv: 1301.4940.

[17] M. Anselmino et al., Eur. Phys. J. A47 (2011) 35, arXiv:1101. 4199.

[18] E.C. Aschenauer et al., Eur. Phys. J. A53 (2017) 71, arXiv:1410.8831.

[19] H. Avakian, B. Parsamyan and A. Prokudin, Riv. Nuovo Cimento 42 (2019) 1.

[20] A. Kotzinian, Nucl. Phys. B441 (1995) 234, arXiv : hep-ph/ 9412283.

[21] P.J. Mulders and R.D. Tangerman, Nucl. Phys. B461 (1996) 197, hep-ph/9510301.

[22] A. Bacchetta et al., JHEP 02 (2007) 093, hep-ph / 0611265.

[23] A. Metz and A. Vossen, Prog. Part. Nucl. Phys. 91 (2016) 136, arXiv: 1607.02521.

[24] D. Boer and P.J. Mulders, Phys. Rev. D57 (1998) 5780, hep-ph / 9711485.

[25] J.C. Collins, Nucl. Phys. B396 (1993) 161, hep-ph/9208213.

[26] A. Signori et al., JHEP 1311 (2013) 194, arXiv:1309.3507.

[27] B. Pasquini, S. Cazzaniga and S. Boffi, Phys. Rev. D78 (2008) 034025, arXiv:hep-ph/0806.2298.

[28] Z. Lu and B.Q. Ma, Nucl. Phys. A741 (2004) 200, hep-ph/0 406171.

[29] M. Anselmino et al., Phys. Rev. D74 (2006) 074015, hep-ph / 0608048.

[30] C. Bourrely, F. Buccella and J. Soffer, Phys.Rev. D83 (2011) 074008, arXiv: 1008.5322.

[31] P. Hagler et al., Europhys. Lett. 88 (2009) 61001, arXiv: hep-lat/0908.1283.

[32] B.U. Musch et al., Phys.Rev. D83 (2011) 094507, arXiv:1011.1213.

[33] M. Wakamatsu, Phys. Rev. D79 (2009) 094028, arXiv: 0903.1886.

[34] P. Schweitzer, M. Strikman and C. Weiss, JHEP 1301 (2013) 163, arXiv: 1210.1267.

[35] M. Anselmino et al., JHEP 1404 (2014) 005, arXiv:1312.6261.

[36] A. Bacchetta et al., JHEP 06 (2017) 081, arXiv:1703.10157.

[37] COMPASS, M. Aghasyan et al., Phys. Rev. D97 (2018) 032006, arXiv: 1709.07374.

[38] M. Aghasyan et al., JHEP 03 (2015) 039, arXiv: 1409.0487.

[39] COMPASS, R. Akhunzyanov et al., arXiv:1802.00584 (2018), arXiv: 1802.00584.

[40] S. Jawalkar et al., Phys. Lett. B782 (2018) 662, arXiv:1709.10054.

[41] HERMES, A. Airapetian et al., Phys. Rev. Lett. 84 (2000) 4047, hep-ex/ 9910062.

[42] HERMES, A. Airapetian et al., Phys. Rev. D64 (2001) 097101, hep-ex/ 0104005.

[43] HERMES, A. Airapetian et al., Phys. Lett. B562 (2003) 182, hep-ex/ 0212039.

[44] HERMES, A. Airapetian et al., Phys. Rev. Lett. 103 (2009) 152002, arXiv: 0906.3918.

[45] COMPASS, C. Adolph et al., Phys. Lett. B744 (2015) 250, arXiv: 1408.4405.

[46] S.M. Aybat and T.C. Rogers, Phys.Rev. D83 (2011) 114042, arXiv:1101. 5057.

[47] COMPASS, C. Adolph et al., Phys. Lett. B770 (2017) 138, arXiv: 1609.07374. 
[48] B. Parsamyan, Int. J. Mod. Phys. Conf. Ser. 40 (2016) 1660029, arXiv: 1504.01599.

[49] COMPASS, B. Parsamyan, PoS QCDEV2015 (2015) 007, arXiv: 1512.06590.

[50] CLAS Collaboration, H. Avakian et al., Phys. Rev. Lett. 105 (2010) 262002, arXiv:hep-ex/1003.4549.

[51] A. Accardi et al., (2012), arXiv:1212.1701.

[52] H.H. Matevosyan et al., Phys. Rev. D92 (2015) 054028, arXiv: 1502.02669.

[53] B. Pasquini and S. Rodini, arXiv:1806.10932 (2018), 1806.10932.

[54] S. Bastami et al., (2018), arXiv:1807.10606.

[55] B. Musch et al., Phys.Rev. D85 (2012) 094510, arXiv:1111. 4249.

[56] M. Engelhardt et al., Phys. Rev. D93 (2016) 054501, arXiv: 1506.07826.

[57] M. Engelhardt, private communications (2018).

[58] CLAS Collaboration, H. Avakian et al., Phys. Rev. D69 (2004) 112004, hep-ex/ 0301005.

[59] HERMES, A. Airapetian et al., Phys. Lett. B648 (2007) 164, hep-ex/ 0612059.

[60] R.N. Cahn, Phys. Lett. B78 (1978) 269.

[61] European Muon, J.J. Aubert et al., Phys. Lett. B130 (1983) 118.

[62] European Muon, M. Arneodo et al., Z. Phys. C34 (1987) 277.

[63] M. Boglione, S. Melis and A. Prokudin, Phys.Rev. D84 (2011) 034033, arXiv: 1106.6177.

[64] HERMES Collaboration, H. Avakian, Nucl. Phys. Proc. Suppl. 79 (1999) 523.

[65] M. Aghasyan et al., Phys. Lett. B704 (2011) 397, arXiv:1106. 2293.

[66] CLAS, W. Gohn et al., Phys. Rev. D89 (2014) 072011, arXiv: 1402.4097.

[67] H. Avakian et al., JLab Experiment E12-06-015. (2008).

[68] M. Radici and A. Bacchetta, Phys. Rev. Lett. 120 (2018) 192001, arXiv: 1802.05212.

[69] J. Czyzewski, Acta Phys.Polon. 27 (1996) 1759, arXiv:hep-ph/9606390.

[70] J.O. Gonzalez-Hernandez et al., Phys. Rev. D98 (2018) 114005, arXiv: 1808.04396.

[71] CLAS, H. Avakian and L. Elouadrhiri, Gerasimov-Drell-Hearn sum rule and its extensions. Proceedings, 3rd International Symposium, GDH 2004, Norfolk, USA, June 2-5, 2004, pp. 228-232, 2004.

[72] A. Bacchetta and M. Radici, Phys. Rev. D69 (2004) 074026, hep-ph/ 0311173.

[73] H. Matevosyan, work in progress (2018).

[74] H.H. Matevosyan, A. Kotzinian and A.W. Thomas, Phys. Rev. Lett. 120 (2018) 252001, arXiv:1712.06384.

[75] M. Anselmino, V. Barone and A. Kotzinian, Phys. Lett. B706 (2011) 46, arXiv: 1109.1132.

[76] CLAS, S. Pisano, EPJ Web Conf. 73 (2014) 02008.

[77] H. Avakian and S. Pisano, PoS DIS2016 (2016) 214.

[78] M. Anselmino, V. Barone and A. Kotzinian, Phys. Lett. B699 (2011) 108, arXiv: 1102.4214. 
[79] W. Melnitchouk and A.W. Thomas, Z. Phys. A353 (1995) 311, arXiv: hep-ph/ 9508205.

[80] M.A. Alberg, J.R. Ellis and D. Kharzeev, Phys. Lett. B356 (1995) 113, arXiv: hep-ph/ 9503333.

[81] J.R. Ellis et al., Phys. Lett. B353 (1995) 319, arXiv: hep-ph/ 9412334.

[82] F. Hautmann et al., arXiv:1408.3015 (2014), arXiv: 1408.3015. 